\title{
Revista de Administração Contemporânea
}

Journal of Contemporary Administration

http://rac.anpad.org.br

Maringá, PR, Brasil, v. 24, n. 1, janeiro/fevereiro, 2020 http://doi.org/10.1590/1982-7849rac2020190380

\section{Editorial Convidado:}

\section{A importância da Ciência Aberta (Open Science) na pesquisa em Administração}

Henrique Castro Martins https://orcid.org/0000-0002-3186-4245 Pontifícia Universidade Católica do Rio de Janeiro, IAG - Escola de Negócios, Rio de Janeiro, RJ, Brasil Editor Associado de Dados Abertos e de Artigos Tutoriais da RAC

“O empreendimento científico é construído sobre uma base de confiança. A sociedade confia que os resultados da pesquisa científica são um reflexo honesto e preciso do trabalho de um pesquisador. Os pesquisadores confiam igualmente que seus colegas reuniram dados cuidadosamente, usaram técnicas estatísticas e analíticas apropriadas, relataram seus resultados com precisão e trataram o trabalho de outros pesquisadores com respeito.” (National Academy of Sciences, 2009, pp. 9, tradução nossa)

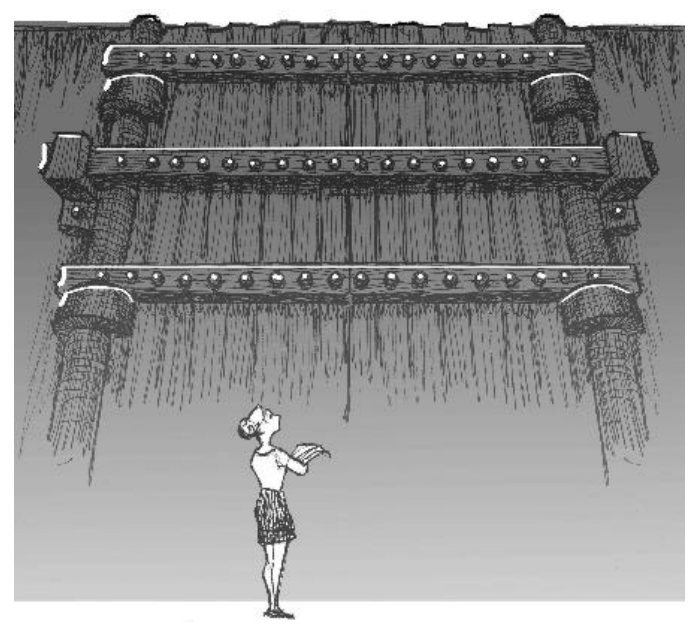

Figura 1. Ilustração de John R. McKiernan (http://whyopenresearch.org/ recuperado em 01 Novembro, 2019) 
Nós todos deveríamos confiar na ciência é um ditado comum entre cientistas, profissionais e a sociedade como um todo. Apesar disso, a ciência está enfrentando uma crise de credibilidade e de confiança (Bergh, Sharp, Aguinis, \& Li, 2017), desde, pelo menos, os anos 2000 (Millstone \& Zwanenberg, 2000). Nos últimos anos, no entanto, temos visto o aumento da discussão de como contra-atacar essa crise de credibilidade (Peng, 2015). Uma ideia que vem tomando destaque nos últimos anos é a de Ciência Aberta (Open Science).

\section{Antes de mais nada, o que é Ciência Aberta (Open Science) (OS)?}

O primeiro aspecto importante de ser discutido sobre OS é definir o seu significado. Até onde conhecemos, não há uma única definição acerca de OS. Contudo, pode-se argumentar que OS é um movimento da comunidade acadêmica de tornar a pesquisa científica (em todo seu ciclo e ambiente) acessível para todos. É um comprometimento vindo dos pesquisadores para com o público (que inclui universidades, instituições financiadoras e outros pesquisadores) para disseminar livre e abertamente os inputs e outputs das pesquisas científicas de uma forma compreensível, acessível e aproveitável, e que permita a reprodutibilidade ${ }^{1}$.

O Movimento de OS começou em 1991 quando o pesquisador Paul Ginsparg criou o repositório virtual arXiv para o compartilhamento de pesquisas em fase de pré-publicação (Open Access, 2019). Em 2002, o movimento cresceu com a Iniciativa de Acesso Livre de Budapeste (https://www.budapestopenaccessinitiative.org/ recuperado em 01 Novembro, 2019) e, em 2003, com a Declaração de Berlin sobre Acesso Livre ao Conhecimento nas Ciências e nas Humanidades (https://openaccess.mpg.de/Berlin-Declaration recuperado em 01 Novembro, 2019). Nos últimos anos, diversas instituições e projetos de suporte à OS foram criados e diversos campos científicos adotaram seus conceitos. Inclusive na nossa área de ciências sociais, já temos um conjunto de stakeholders clamando por mais transparência em pesquisas (Aguinis, Cascio, \& Ramani, 2017; Mendes-Da-Silva, 2019; Miguel et al., 2014).

\section{O que OS envolve?}

OS envolve um conjunto de práticas e conceitos baseados na ideia geral de tornar a ciência mais transparente e acessível a todos. O conceito mais conhecido é o de Acesso Livre (Open Access), que consiste na prática de tornar os artigos publicados acessíveis sem restrições. Mais recentemente, outros conceitos vêm sendo trazidos a debate, como por exemplo, Pré-registro de pesquisa (i.e., Preregistration) e Revisão dos Pares Aberta (Open Peer Review). Abaixo, oferece-se um breve resumo dessas práticas.

Acesso Livre (Open Access): é o acesso virtual, sem restrição (incluindo restrições financeiras), a todo artigo científico publicado em periódicos acadêmicos. Existem duas formas diferentes de Acesso Livre. Acesso Livre Ouro (Golden open access) se refere ao acesso irrestrito em todas as suas formas. Nesse modo, os custos de publicação são arcados pelos autores, ao invés de pelos leitores. Acesso Livre Verde (Green open access) se refere a publicações que só podem ser acessadas irrestritamente após um período de embargo. Durante o período de embargo, a publicação só pode ser acessada via compartilhamento pelos autores.

Dados e Materiais Abertos (Open Data and Open Materials): se refere à possibilidade de acessar livremente, sem restrições técnicas ou legais, todos os dados e demais materiais utilizados em uma pesquisa científica. O público pode acessar e reutilizar os materiais sem precisar de consenso dos autores. Isso permite que outros pesquisadores estendam a pesquisa original, testem diferentes hipóteses e reutilizem os dados em diferentes pesquisas.

Código Aberto (Open Source): se refere a software e pacotes de códigos disponíveis de forma livre a todo o público, sem restrições legais ou técnicas. Softwares de código aberto podem ser customizados e alterados pelo usuário de forma livre e sem limitações de direitos autorais.

Avaliação Pelos Pares Aberta (Open Peer Review): é uma variação da avaliação pelos pares tradicional. Um artigo, tipicamente, é publicado após a avaliação pelos pares, que geralmente é feita de forma anônima (i.e., 
autores e avaliadores não conhecem a identidade um do outro). A avaliação pelos pares aberta torna a identidade de todos os participantes disponível a todos. Adicionalmente, os comentários, sugestões dos avaliadores e as respostas dos autores são disponibilizadas como material suplementar ao artigo publicado. Consequentemente, autores, revisores e os leitores conhecem e podem avaliar o histórico de revisão do artigo ao lado da versão final publicada (Ross-Hellauer, 2017).

Pré-registro de pesquisa (Preregistration of research): é o comprometimento feito pelos autores de seguir de forma estrita um determinado protocolo de pesquisa, definido antes da coleta dos dados necessários. $\mathrm{O}$ benefício principal é tornar o processo de geração de hipóteses independente do processo de coleta dos dados e avaliação dos resultados. Isso aumenta a transparência das escolhas dos autores e diminui a possibilidade de harking (hypothesizing after the results are known). Pesquisas pré-registradas podem ser submetidas, avaliadas pelos pares, e aceitas para publicação, independentemente da posterior confirmação ou não das hipóteses. Nesses casos, os periódicos se comprometem a publicar a pesquisa pré-registrada final, independente dos seus resultados.

\section{Quais são os benefícios da OS?}

Existem diversos argumentos a favor da OS. Há benefícios para virtualmente todos os participantes da comunidade científica e para o público em geral. Para pesquisadores, OS aumenta a visibilidade das suas pesquisas, o seu reconhecimento pessoal, sua reputação e sua credibilidade; aumenta a possibilidade de encontrar novas parcerias de pesquisa, a probabilidade de obter financiamentos, a probabilidade de que o público em geral acessará sua pesquisa e, potencialmente, aumenta o número de citações e atenção por parte da mídia. Para a comunidade científica, diminui os custos operacionais de coleta, tabulação e manipulação de dados de pesquisas, aumenta a colaboração entre agentes que coletam dados e agentes que utilizam dados, aumenta a possibilidade de se replicar pesquisas publicadas e auxilia a elaboração de materiais didáticos de pesquisa. Para financiadores de pesquisa, ajuda a entender e a controlar a alocação dos recursos, públicos e privados, destinados à pesquisa e avaliar o seu retorno econômico; também diminui a probabilidade de gastos em pesquisas ou experimentos duplicados ou muito semelhantes. Finalmente, para o público em geral, ajuda os agentes decisores a formular melhores e mais eficientes políticas públicas e a melhorar o debate político; além disso, aumenta o direito democrático de acesso a dados e conhecimento por parte de todos os participantes da sociedade, o que permite maior engajamento do público na atividade científica (Aguinis et al., 2017; McKiernan et al., 2016; Pampel \& Dallmeier-Tiessen, 2014; Piwowar \& Vision, 2013).

\section{Quem está envolvido com OS?}

Diversas instituições e agentes estão envolvidos no movimento OS. A comunidade OS inclui organizações supranacionais, como a União Europeia e a OECD, organizações privadas, universidades, periódicos acadêmicos, entre outros. Uma breve lista desses agentes se encontra a seguir:

União Europeia (EU): EU, através do seu braço executivo, a Comissão Europeia, divulgou diversos documentos e recomendações acerca de OS. Por exemplo, os documentos Towards better access to scientific information: Boosting the benefits of public investments in research (European Union [EU], 2012) $\mathrm{e}$ Recommendation on access to and preservation of scientific information (European Union [EU], 2018).

OECD (Organização para Cooperação e Desenvolvimento Econômico): OECD suporta OS e possui um reporte completo em que explica questões relacionadas à OS (Organization for Economic Co-operation and Development [OECD], 2015). O objetivo da OECD é promover a OS e avaliar o seu impacto no desenvolvimento da pesquisa e inovação.

FOSTER: é um projeto de 2 anos financiado pela EU, que conta com a participação de 11 parceiros de 6 países europeus. O objetivo do projeto é contribuir para a mudança de comportamento dos pesquisadores da EU de forma a assegurar que OS se torne a prática vigente na comunidade científica (https://www.fosteropenscience.eu recuperado em 01 Novembro, 2019). 
F1000Research: é uma plataforma para cientistas e pesquisadores que publica, de forma imediata, artigos e materiais derivados de pesquisa. Todos os materiais se beneficiam do processo de Avaliação Pelos Pares Aberta e todo material de pesquisa dos artigos publicados é disponibilizado livremente (https://f1000research.com recuperado em 01 Novembro, 2019).

Data in Brief: é um periódico acadêmico, de acesso livre, que publica artigos com bases de dados. Esses artigos descrevem uma base de dados coletada pelos pesquisadores para ser usada em uma pesquisa científica ou para ser disponibilizada aos pares. Os dados são disponibilizados livremente para todo o público (https://www.journals.elsevier.com/data-in-brief recuperado em 01 Novembro, 2019).

Center for Open Science: é uma organização cuja missão é aumentar a transparência, integridade e reprodutibilidade das pesquisas científicas. A organização oferece diversos mecanismos e produtos relacionados à OS, como por exemplo, pré-registro de pesquisa e financiamento coletivo de coleta de dados, e diversas iniciativas que aumentam reprodutibilidade (https://cos.io/ recuperado em 01 Novembro, 2019).

Periódicos acadêmicos: ao fim de 2019, diversos periódicos acadêmicos já apresentam normas e orientações relacionadas à OS. Geralmente, os periódicos se incluem em um dos grupos a seguir: (a) Encoraja disponibilização de dados, (b) Encoraja a disponibilização de dados e requer declaração dos autores acerca da sua decisão, (c) "Requer disponibilização de dados, ou (d) Requer disponibilização de dados com avaliação por pares". Nas áreas de Administração, Contabilidade, Finanças e Estratégia, o mais comum, atualmente, é o periódico se enquadrar na opção A ou B.

Uma lista não exaustiva de periódicos que tem orientações acerca de OS inclui:

Finance and Stochastics;

- International Entrepreneurship and Management Journal;

. Journal of Corporate Finance;

. Journal of Economics and Finance;

. Journal of Management \& Governance;

- Operations Management Research;

- Review of Accounting Studies;

. Review of Quantitative Finance and Accounting.

\section{Quais são os argumentos contrários à OS?}

Um dos pilares essenciais da OS é transparência. Contudo, o aumento de transparência sempre leva a novas demandas por mais transparência, de tal forma que precisamos nos perguntar: qual o nível ótimo de transparência na ciência? Todos os tipos de transparência são desejáveis? Quanto de transparência é o suficiente?

Por exemplo, considere a Avaliação Pelos Pares Aberta, em que autores e revisores sabem suas respectivas identidades. Certamente, isso aumenta a transparência nas revisões. Contudo, nesse sistema, é possível que revisores escrevam pareceres menos críticos ou com mais parcimônia que o ideal. Além disso, os autores podem ter interesse em focar na resolução de sugestões feitas por revisores com maior reputação ou mais status na área de pesquisa, ao invés de focar nos comentários mais necessários e importantes ao desenvolvimento da pesquisa. No limite, Avaliações Pelos Pares Aberta malfeitas podem levar a revisões piores e afetar a qualidade dos artigos publicados.

Adicionalmente, a disponibilização de dados e materiais de pesquisa pode incentivar o comportamento antiético e aumentar o debate com base em ideologias. Considere a situação em que os dados de uma pesquisa, cujos resultados são contrários a um grupo de interesse específico, são livremente disponibilizados. Isso pode provocar uma caça a cientistas por esses grupos de interesse, como descrito por Camarata (2019). No limite, esse tipo de comportamento pode prejudicar o debate político de um determinado tema e afetar a própria democracia. 
Além disso, permitir que não-especialistas e não-cientistas tenham acesso a dados de pesquisa pode levar a conclusões indevidas devido a análises inadequadas de dados. Isso é, usuários de dados científicos devem sempre se comprometer a usá-los de forma competente e dentro de um contexto institucional baseado em prestação de contas (i.e., accountability). Na medida em que os dados abertos são utilizados livremente em um ambiente sem controle por parte do cientista, a análise dos dados pode ficar comprometida ou ser feita de forma equivocada, o que pode criar efeitos negativos para a própria pesquisa.

Finalmente, comunicações e exposições de pesquisas em formatos não-científico, geralmente, não exploram a totalidade da complexidade e das incertezas de uma pesquisa. Com o objetivo de aumentar a compreensão, a comunicação sacrifica precisão e complexidade. Isso pode levar a interpretações errôneas da pesquisa e a críticas ao processo científico.

Todos esses exemplos são argumentos contrários à OS e que podem levar a ineficiências ou a resultados indesejados. De modo geral, precisa-se de um sistema que, simultaneamente, aumente a transparência das pesquisas sem comprometer a liberdade individual dos cientistas e sem incentivar comportamentos antiéticos por parte de não-cientistas.

\section{Como a RAC está incentivando OS?}

Tipicamente, autores no passado recente, utilizaram a Pesquisa Fechada e, apesar disso, conseguiram criar boa reputação com base em trabalho científico qualificado. Assim, uma mudança cultural é necessária para permitir que OS seja uma realidade. Todos nós precisaremos mudar hábitos antigos para auxiliar o desenvolvimento da OS. Talvez nossa primeira grande ação deva ser discutir OS com nossos estudantes e fazer uma autorreflexão de como podemos contribuir para o movimento.

Adicionalmente, é bastante comum que periódicos cobrem taxas para disponibilizar materiais de forma livre (i.e., Golden Open Access) que podem chegar a USD 1.000, USD 1.500, ou até mesmo valores maiores. Mesmo o Acesso Livre Verde (Green Open Access) é possível somente após um período de embargo que pode chegar a 24 meses. Essas são importantes barreiras à OS. Dessa forma, Editoras precisam também rever estratégias de comercialização e rentabilização de materiais de pesquisa.

A equipe editorial da RAC acredita, no entanto, que OS é um passo para o futuro necessário à pesquisa acadêmica e incentiva o compartilhamento aberto de dados e demais materiais de pesquisa. Entende-se que, às vezes, dados de pesquisa não podem ser livremente disponibilizados devido a acordos e normas legais (por exemplo, entrevistas com menores de idade ou com fontes com contrato de anonimato). Nesses casos, a RAC tem convidado que os autores façam uma declaração explicando os pontos que impedem a disponibilização de materiais. No entanto, se o artigo contém uma parte de dados que pode ser disponibilizada (exemplo, dados de fontes públicas ou dados previamente livres à comunidade), a RAC convida que os autores disponibilizem essa parte e omita a parte cuja disponibilização não é possível. Além disso, a RAC encoraja que os autores compartilhem livremente todo material de programação e análise (como do-files, r-scripts etc.) bem como todo material adicional que possa ser compartilhado.

Desde 2018, a equipe editorial da RAC guia os autores de artigos aceitos a utilizarem repositórios virtuais (por exemplo, Zenodo ou Mendeley) para disponibilização de seus materiais de pesquisa. Esses repositórios criam uma referência para os arquivos (e um código DOI permanente) que pode ser citada em trabalhos futuros. Para aumentar o acesso pela comunidade e a visibilidade dos autores, a RAC inclui, no artigo publicado, a referência dos materiais e dados disponibilizados pelos autores. Além disso, a RAC providencia e inclui no artigo publicado um código QR ligado aos materiais e dados disponibilizados. Isso auxilia a utilização de smartphones e tablets para o gerenciamento das referências por parte dos nossos leitores. Ademais, da mesma forma que diversos periódicos internacionais, a RAC utiliza o repositório público Dataverse, criado e financiado por Harvard, para depositar materiais dos artigos publicados. O endereço eletrônico do perfil da RAC nesse repositório pode ser encontrado em: 〈https://dataverse.harvard.edu/dataverse/rac $>$. Isso torna disponível os dados de autores para a comunidade acadêmica internacional, aumentando a visibilidade e o potencial de citação dos artigos da RAC. 
De modo geral, a RAC está comprometida em aumentar a visibilidade de nossos autores e incentivar aspectos relacionados à OS. Acreditamos que a disponibilização de materiais e dados abertos, em particular, e a OS, em geral, tem o potencial de aumentar significativamente o alcance de nossas pesquisas, aumentar o potencial de citação de impacto de nossos artigos, e facilitar o debate e o relacionamento entre pesquisadores e praticantes.

Finalmente, eu gostaria de mencionar que, ao fim de 2019, eu me juntei à equipe editorial da RAC como o seu primeiro Editor de Tutoriais e Dados Abertos. Meu objetivo principal é discutir os conceitos de OS e advogar pelos seus benefícios para todos os agentes interessados em pesquisa acadêmica. Além disso, terei o prazer de iniciar uma nova seção da RAC, que passará a publicar artigos-tutoriais que sejam um guia empírico e que ofereçam um framework para pesquisas quantitativas e qualitativas.

É, ao mesmo tempo, um prazer e um desafio me juntar a um dos mais prestigiados periódicos acadêmicos de Administração do Brasil. Eu tenho confiança de que, ao debater OS, nós conseguiremos expandir o movimento aos nossos leitores e incentivar a criação de um ambiente de pesquisa e de uma comunidade científica mais inclusiva, transparente e eficiente.

\section{Nota}

${ }^{1}$ No relatório da OECD, Making Open Science a Reality (Tornando a Ciência Aberta uma Realidade, tradução nossa) (OECD, 2015), o termo refere-se à esforços de pesquisadores, governos, agências de fomento à pesquisa ou a própria comunidade científica para que os principais resultados de pesquisa com financiamento público publicações e dados da pesquisa - sejam acessíveis ao público em formato digital, com ou sem restrição mínima, como forma de acelerar a pesquisa. Esses esforços são do interesse de aumentar a transparência e a colaboração, e promover a inovação.

\section{Referências}

Aguinis, H., Cascio, W. F., \& Ramani, R. S. (2017). Science's reproducibility and replicability crisis: International business is not immune. Journal of International Business Studies, 48(6), pp. 653-663. https://doi.org/10.1057/s41267-017-0081-0

Bergh, D. D., Sharp, B. M., Aguinis, H., \& Li, M. (2017). Is there a credibility crisis in strategic management research? Evidence on the reproducibility of study findings. Strategic Organization, 15(3), 423-436. https://doi.org/10.1177/1476127017701076

Camarata, S. (2019). The dark side of social media activism in science. Retrieved in November 01, 2019, from https://www.psychologytoday.com/us/blog/the-intuitive-parent/201907/the-dark-side-social-mediaactivism-in-science.

European Union [EU] (2012). Towards better access to scientific information: Boosting the benefits of public investments in research. Retrieved in October 30, 2019, from https://ec.europa.eu/research/sciencesociety/document library/pdf 06/era-communication-towards-better-access-to-scientificinformation_en.pdf.

European Union [EU] (2018). Recommendations on access to and preservation of scientific information. Retrieved in 30 October 2019, from https://eur-lex.europa.eu/legal-content/EN/TXT/?uri=CELEX:32018H0790.

McKiernan, E. C., Bourne, P. E., Brown, C. T., Buck, S., Kenall, A., Lin, J., McDougall, D., Nosek, B. A., Ram, K., Soderberg, C. K., Spies, J. R., Thaney, K., Updegrove, A., Woo, K. H., \& Yarkoni, T. (2016). How open science helps researchers succeed. ELife, 5, 1-19. https://doi.org/10.7554/eLife.16800 
Mendes-Da-Silva, W. (2019). Temos sido transparentes o suficiente? Desafios à replicabilidade e à credibilidade da pesquisa na área de negócios. Revista de Administração Contemporânea, 23(5). https://doi.org/10.1590/1982-7849rac2019190306

Miguel, E., Camerer, C., Casey, K., Cohen, J., Esterling, K. M., Gerber, A., Glennerster, R., Green, D. P., Humphreys, M., Imbens, G., Laitin, D., Madon, T., Nelson, L., Nosek, B. A., Petersen, M., Sedlmayr, R., Simmons, J. P., Simonsohn, U., \& Van Der Laan, M. (2014). Promoting transparency in social science research. Science, 343(6166), 30-31. https://doi.org/10.1126/science.1245317

Millstone, E., \& Zwanenberg, P. van (2000). A crisis of trust: For science, scientists or for institutions? Nature Medicine, 6, 1307-1308. https://doi.org/10.1038/82102

National Academy of Sciences. (2009). On being a scientist: A guide to responsible conduct in research (3rd ed.). Washington (DC): National Academies Press (US). https://doi.org/10.17226/12192

Organization for Economic Co-operation and Development [OECD]. (2015). Making open science a reality. OECD Science, Technology and Industry Policy Papers, (25). https://doi.org/10.1787/5jrs2f963zs1-en

Open Access (2019). Origins of the open access movement. Retrieved in October 30, 2019, from https://openaccess.net/en/information-on-open-access/history-of-the-open-access-movement

Pampel, H., \& Dallmeier-Tiessen, S. (2014). Open research data: From vision to practice. In Bartling S., Friesike S. (eds), Opening Science (pp. 213-224). Cham, Switzerland: Springer. https://doi.org/10.1007/978-3319-00026-8_14

Peng, R. (2015). The reproducibility crisis in science: A statistical counterattack. Significance, 12(3), 30-32. https://doi.org/10.1111/j.1740-9713.2015.00827.x

Piwowar, H. A., \& Vision, T. J. (2013). Data reuse and the open data citation advantage. PeerJ, 2013(1), e175. https://doi.org/10.7717/peerj.175

Ross-Hellauer, T. (2017). What is open peer review? A systematic review [version 2; peer review: 4 approved]. F1000Research, 6. https://doi.org/10.12688/f1000research.11369.2

\author{
Autor \\ Henrique Castro Martins \\ Rua Marquês de São Vicente, $n^{\circ}$. 255, 22451-900, Rio de Janeiro, RJ, Brasil \\ E-mail: $\underline{\text { hcm@iag.puc-rio.br }}$
}

\title{
Corpo Editorial Científico e Equipe Editorial para esta edição:
}

\section{Conselho Editorial}

Anielson Barbosa da Silva (UFPB, João Pessoa, Brasil)

Antonio Carlos Gastaud Maçada (UFRGS, Porto Alegre, Brasil)

Ely Laureano Paiva (FGV, São Paulo, Brasil)

Fabio Vizeu Ferreira (UP, Curitiba, Brasil)

Maria José Tonelli (FGV, São Paulo, Brasil)

Rogério Hermida Quintella (NPGA/UFBA, Salvador, Brasil)

Valmir Emil Hoffmann (UnB, Brasília, Brasil)

Wesley Mendes-da-Silva (EAESP/FGV, São Paulo, Brasil)

\section{Editor-chefe}

Wesley Mendes-da-Silva (EAESP/FGV, São Paulo, Brasil)

\section{Editores Associados}

André Luiz Maranhão de Souza-Leão (UFPE, Recife, Brasil) 
Fabio Caldieraro (EAESP/FGV, São Paulo, Brasil)

Gilnei Luiz de Moura (UFSM, Santa Maria, Brasil)

Henrique Castro Martins (IAG PUC-Rio, Rio de Janeiro, Brasil)

Ismael Ali Ali (Kent State University, Ohio, EUA)

Marcus Cunha Junior (University of Georgia, EUA)

Paulo César Matui (UniGranRio, Rio de Janeiro, Brasil)

Samy Dana (FGV/EAESP, São Paulo, Brasil)

Victor Manoel Cunha de Almeida (COPPEAD/UFRJ, Rio de Janeiro, Brasil)

\section{Corpo Editorial Científico}

Aureliano Angel Bressan (CEPEAD/UFMG, Belo Horizonte, Brasil)

Bryan Husted (York University, Canadá)

Carlos M. Rodriguez (Delaware State University, EUA)

Cristiana Cerqueira Leal (Universidade do Minho, Portugal)

Diógenes de Souza Bido (Mackenzie, São Paulo, Brasil)

Elin Merethe Oftedal (University of Troms $\varnothing$, Noruega)

Emilio Jose Monteiro Arruda Filho (Unama, Belém, Brasil)

Fábio Frezatti (FEA/USP, São Paulo, Brasil)

Felipe Monteiro (Wharton/University of Pennsylvania, EUA)

Howard J. Rush (University of Brighton, Reino Unido)

James Robert Moon Junior (Georgia Institute of Technology, EUA)

John L. Campbell (University of Georgia, EUA)

José Antônio Puppim de Oliveira (United Nations University, Yokohama, Japão)

Julián Cárdenas (Freie Universität, Berlin, Alemanha)

Lucas Barros (EAESP/FGV, São Paulo, Brasil)

Luciano Rossoni (UniGranRio, Rio de Janeiro, Brasil)

M. Philippe Protin (Université Grenoble Alpes, França)

Paulo Estevão Cruvinel (Embrapa Instrumentação, São Carlos, Brasil)

Rodrigo Bandeira de Mello (Merrimack College, EUA)

Rodrigo Verdi (MIT Massachusetts Institute of Technology, Cambridge, EUA)

Valter Afonso Vieira, (UEM, Maringá, Brasil)

Wagner Kamakura (Jones Graduate School of Business, Rice University, Houston, EUA)

\section{Editoração}

Diagramação e Normas da APA: Kler Godoy (ANPAD, Maringá, Brasil)

Periodicidade: Bimestral

Circulação: Acesso totalmente gratuito 